\title{
Discerning Between Macular Hemorrhages Due to Macular Neovascularization or Due to Spontaneous Bruch's Membrane Rupture in High Myopia: A Comparative Analysis Between OCTA and Fluorescein Angiography
}

\author{
Marco Battista - Riccardo Sacconi - Enrico Borrelli • Anna Crepaldi • \\ Federico Fantaguzzi · Eliana Costanzo • Daniele De Geronimo · \\ Mariacristina Parravano · Francesco Bandello · Giuseppe Querques \\ Received: January 3, 2022 / Accepted: February 7, 2022 / Published online: February 20, 2022 \\ (C) The Author(s) 2022
}

\section{ABSTRACT}

Introduction: To evaluate the sensitivity and specificity of optical coherence tomography angiography (OCTA) in comparison to fluorescein angiography (FA) in discerning between macular hemorrhages due to myopic macular neovascularization (m-MNV) and idiopathic macular hemorrhage (IMH) in patients with high myopia (HM).

Methods: In this retrospective study, 14 eyes of 14 patients (mean age $60 \pm 16$ years) affected by macular hemorrhage due to $\mathrm{HM}$ were

Marco Battista and Riccardo Sacconi contributed equally to this study and should be considered equivalent authors.

M. Battista - R. Sacconi - E. Borrelli - A. Crepaldi · F. Fantaguzzi - F. Bandello - G. Querques School of Medicine, Vita-Salute San Raffaele University, Milan, Italy

M. Battista - R. Sacconi - E. Borrelli - A. Crepaldi · F. Fantaguzzi · F. Bandello · G. Querques Division of Head and Neck, Ophthalmology Unit, IRCCS San Raffaele Scientific Institute, Milan, Italy

E. Costanzo - D. De Geronimo - M. Parravano Foundation G.B. Bietti-IRCCS, Rome, Italy

G. Querques $(\bowtie)$

Department of Ophthalmology, University Vita-

Salute San Raffaele, Via Olgettina 60, Milan, Italy

e-mail: giuseppe.querques@hotmail.it;

giuseppe.querques@unisr.it included. All patients underwent OCTA and FA at the time of macular hemorrhage (i.e., baseline) and were followed for a 3-month followup.

Results: By means of FA, 8 out of 14 eyes with macular hemorrhage (57\%) were diagnosed as type $2 \mathrm{~m}-\mathrm{MNV}$, whereas 6 eyes (43\%) were diagnosed as IMH. Interestingly, OCTA displayed the presence of a neovascular network in all cases previously diagnosed as m-MNV using FA, and also excluded the presence of anomalous flow in all IMH eyes. This accounted for the high sensitivity and specificity of OCTA for m-MNV detection in HM cases with macular hemorrhage. After 3-month follow-up, BCVA improved from $0.39 \pm 0.15$ to $0.21 \pm 0.14 \log$ MAR $(p=0.006)$ in patients with m-MNV treated by a mean of $2.3 \pm 0.9$ intravitreal anti-VEGF injections. Conversely, BCVA improved without treatment (from $0.55 \pm 0.48$ to $0.17 \pm 0.08 \log$ MAR, $p=0.112$ ) in patients with IMH.

Conclusions: OCTA is able to differentiate with excellent reliability between the presence of m-MNV in patients with HM presenting with a new macular hemorrhage and an IMH. This could be of paramount relevance in the clinical setting for the diagnosis and treatment of patients with HM.

Keywords: High myopia; Idiopathic macular hemorrhages; Myopic macular 
neovascularization; Optical coherence tomography-angiography

\section{Key Summary Points}

What was known before?

Myopic macular neovascularization (mMNV), usually revealing itself as a macular hemorrhage, is a common complication in high myopia resulting in poor visual outcome, if left untreated.

Distinguishing macular hemorrhages due to myopic neovascularization from idiopathic macular hemorrhages in high myopic eyes can be challenging in clinical practice.

Fluorescein angiography (FA) is nowadays the gold standard to detect $\mathrm{m}-\mathrm{MNV}$; however, its invasiveness and the masking effect exerted by dense hemorrhages should be considered.

\section{What this study adds}

Optical coherence tomography angiography (OCTA) could be regarded as an excellent modality in identifying a neovascular pattern in cases of myopic hemorrhages, thus differentiating a neovascularization from an idiopathic macular hemorrhage with high sensitivity and specificity.

OCTA proved to be a reliable alternative to fluorescein angiography in the management of myopic macular hemorrhages.

\section{INTRODUCTION}

Myopia is a common visual disorder whose prevalence is increasing worldwide. It was estimated to affect up to 2.5 billion people by 2020 , with $27-33 \%$ of these occurring as high myopia (HM) [axial length $>25.5 \mathrm{~mm}$ or refractive error $>6$ diopters (D)] [1]. Myopic maculopathy (MM) was firs reported by Curtin and Karlin in 1970 and included features as chorioretinal atrophy, lacquer cracks, Fuch's spot, posterior staphyloma, and optic disc changes in eyes affected by HM [2]. Classifications of MM evolved through the years, but the initial characteristics of chorioretinal atrophy, worsening with the increase of axial length, remain in the more modern and simplified classification [3]. Myopic macular neovascularization ( $\mathrm{m}-\mathrm{MNV}$ ) is a significant sight-threatening complication in $\mathrm{MM}$, often associated with macular hemorrhage at presentation, and prompt diagnosis and treatment are mandatory to avoid consistent loss of visual acuity [4]. Lacquer cracks seem to be associated with the onset of $\mathrm{m}-\mathrm{MNV}$ since most of the neovessels branch from a lacquer crack and vascular endothelial growth factor (VEGF) is one of the main actors in m-MNV development [5]. A causal association between lacquer cracks and the site of scleral expansion crossed by a perforating vessel has recently been hypothesized [6].

Conversely, idiopathic macular hemorrhage (IMH) is another common complication in MM eyes [7]. IMHs result in a localized bleeding initially confined in the subretinal space. The formation of new lacquer crack can be associated with IMH. In this regard, perforating scleral vessels could be the trigger for lacquer cracks formation: the scleral expansion exerted by their blood flow is the primary factor in the tissue remodeling [6]. If the amount of the subretinal hemorrhage is consistent, Bruch's membrane and retinal pigmented epithelium (RPE) could stretch and rupture in a weaker point, enabling blood to pass through the RPE into the subretinal space. The distinction between an IMH located above the RPE and a macular hemorrhage secondary to $\mathrm{m}-\mathrm{MNV}$ can be challenging [8].

Fluorescein angiography (FA) is ordinarily the most useful imaging method to assess the presence of $\mathrm{m}-\mathrm{MNV}$, as it usually manifests as a type $2 \mathrm{MNV}$ [9]. However, FA is an invasive procedure burdened by side effects such as nausea, dizziness, allergic reactions up to anaphylactic shock, and it could be of limited value 
in large macular hemorrhages exerting a masking effect [10].

Optical coherence tomography angiography (OCTA) is a relatively new non-invasive imaging technique [11]. OCTA can successfully be applied in the diagnosis and follow-up of MNV in different diseases [12-16]. Recent studies found a high sensitivity of OCTA (86.5\%), compared to FA, in detecting macular neovascularization in age-related macular degeneration [17].

The aim of our study is to evaluate the sensitivity and specificity of OCTA in comparison to FA in discerning between macular hemorrhages due to m-MNV and simple bleeding due to spontaneous Bruch's membrane rupture in patients with HM (i.e., IMH). This could be of paramount relevance in the clinical setting for the diagnosis and treatment of patients with HM.

\section{METHODS}

In this retrospective longitudinal study, subjects 18 years of age and older affected by MM with a newly diagnosed macular hemorrhage in at least one eye were identified from the medical records of the Medical Retina and Imaging Unit at the San Raffaele Scientific Institute, Milan, Italy and G. B. Bietti Foundation, Rome, Italy. This study adhered to the 1964 Helsinki declaration and its later amendments. All individual participants included in the study signed the informed consent that was previously approved by the local ethics committee.

All the records between January 2018 and November 2020 were analyzed.

Diagnosis of macular hemorrhage was determined by fundus examination, and documented by multicolor imaging or color fundus image. Structural OCT, FA, and OCTA scans performed on the same day of the onset visit were required as primary inclusion criteria. The macular surface was considered as a circular area of $5.5 \mathrm{~mm}$ diameter centered on the fovea and located within the temporal vascular branches. The area corresponds to the entire extension of the EDTRS macular grid. Hemorrhages outside the macular area were excluded from the analysis.

Exclusion criteria included (1) evidence of myopic traction; (2) evidence of posterior inflammatory disorders, as multifocal choroiditis and punctate inner choroidopathy, recognized to be potential causes of MNV in myopic eyes [18]; (3) history of any ocular surgery 6 months before the onset visit; (4) any previous retinal treatments (e.g., intravitreal injections, photodynamic therapy) excluding peripheral barrage laser; (5) history or evidence of other retinal and optic nerve diseases; (6) poor quality of images.

All subjects had multicolor imaging and/or color fundus imaging, structural spectral-domain OCT, FA, OCTA, and axial length (AL) measurement. True-color fundus images were acquired through the EIDON device (CenterVue, Padua, Italy); multicolor imaging, structural OCT, and FA were performed with the Heidelberg Spectralis HRA + OCT device (Heidelberg Engineering, Heidelberg, Germany). A minimum signal strength of 25 was required for the OCT images to be included, as recommended by the manufacturer [19]. OCTA scans were obtained with PLEX Elite 9000 (Carl Zeiss Meditec Inc., Dublin, CA, USA). AL measurements were performed using the IOLMaster device (Carl Zeiss Meditec AG, Jena, Germany). In addition, data from enrolled patients, including demographic information, measurement of best-corrected visual acuity (BCVA) using Snellen charts then converted to logarithm of the minimum angle of resolution ( $\log$ MAR) for statistical analysis, and dilated fundus examination were obtained. Finally, clinical data and multimodal imaging evaluation after 3-month follow-up were evaluated.

\section{Imaging Analysis}

Two independent and experienced readers (MB and AC) first reviewed multicolor, structural OCT, OCTA, and FA images for eligibility. Eyes not fulfilling the study inclusion and exclusion criteria were excluded. Eligible eyes were independently graded by two experienced readers (RS and EB) for the assessment of the 

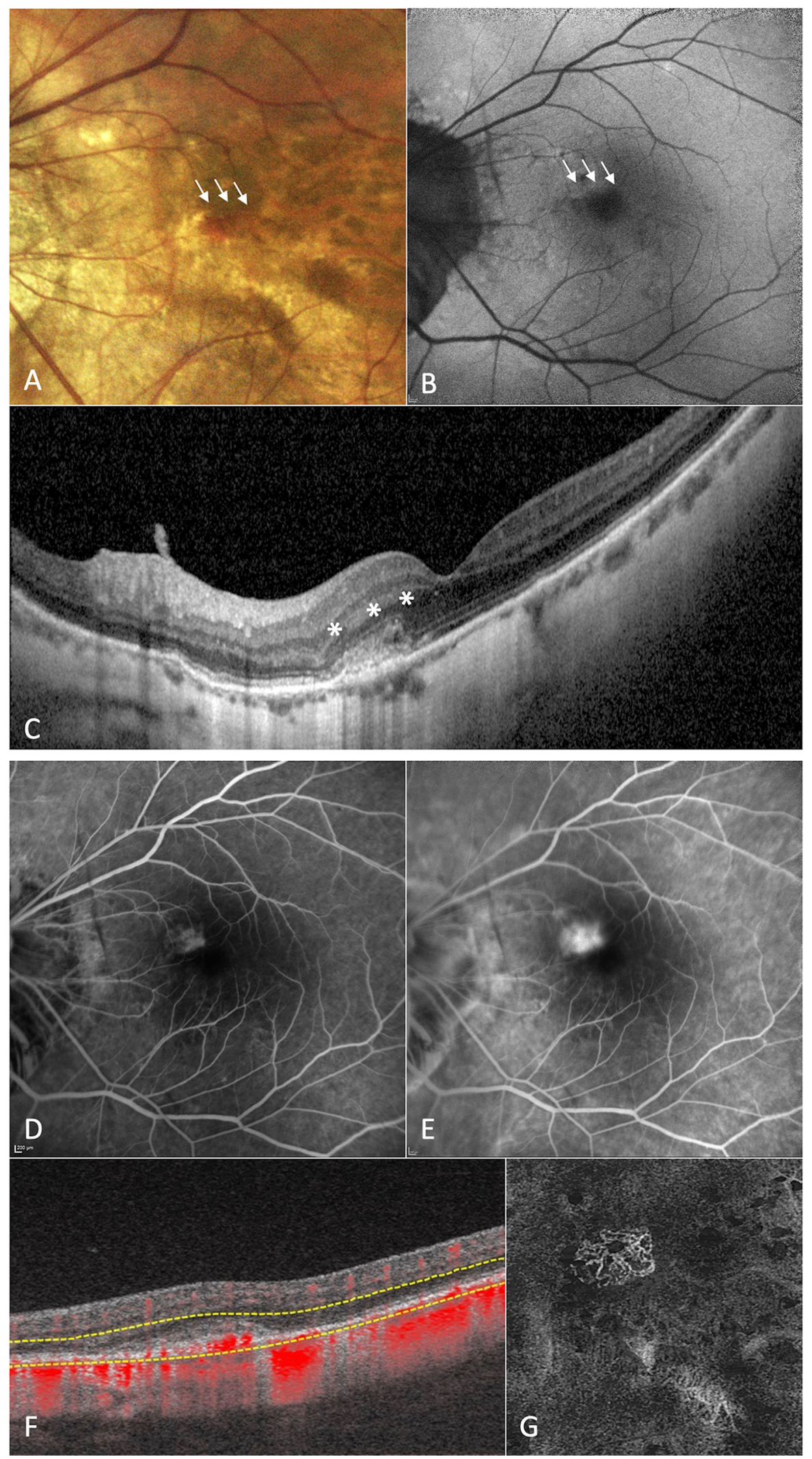
4Fig. 1 Multimodal retinal imaging of a patient with high myopia affected by macular hemorrhage due to type 2 macular neovascularization (MNV) in the left eye. Fundus imaging (a) and fundus autofluorescence (b) showing the presence of a macular hemorrhage in the parafoveal area (white arrows). Structural optical coherence tomography (OCT) passing through the lesion (c) illustrating the presence of subretinal hyperreflective material (SHRM) above the retinal pigment epithelium with small subretinal hyporeflective exudation (asterisks). Fluorescein angiography displaying a well-defined hyperfluorescent lesion in the early angiographic phases (d) with moderate leakage in the late phases (e), matching with a diagnosis of type $2 \mathrm{MNV}$ in the site of the macular hemorrhage. $6 \times 6 \mathrm{~mm}$ en-face OCT-angiography image $(\mathbf{g})$ and corresponding B-scans with flow (f) confirming the presence of a neovascular network in the SHRM location

characteristic of the myopic macular hemorrhage. More specifically, the two graders, masked to each other and to patients, independently assessed the presence/absence of m-MNV underneath the macular hemorrhage on FA images, and, independently, on OCTA scans. The OCTA analysis was performed on three distinct PLEX Elite 9000 slabs: the outer retina to choriocapillaris (ORCC), the avascular zone (from the outer boundary of the OPL to $99 \mu \mathrm{m}$ above Bruch's membrane), and the choriocapillaris. Discrepancies between graders were resolved by additional discussion and open adjudication to yield a single assessment for each case. The final decision in those cases in which the two graders did not agree on a single consensus result was made by the senior author (GQ). No post-acquisition manual segmentation was needed to improve the angiographic images.

If $\mathrm{m}-\mathrm{MNV}$ was not detected by both FA and OCTA, the hemorrhage was considered as IMH. If $\mathrm{m}-\mathrm{MNV}$ was detected by both FA and OCTA, the hemorrhage was considered as m-MNV. In the case of discrepancy between FA and OCTA, the gold standard for the presence of $\mathrm{m}-\mathrm{MNV}$ was considered the FA examination, as reported by the literature [19].

The primary outcome measure was to assess the sensitivity and specificity of OCT-A in discerning between $\mathrm{m}-\mathrm{MNV}$ and $\mathrm{IMH}$, in comparison to FA.

\section{Statistical Analysis}

Statistical analyses were performed using SPSS Statistics Version 20 (IBM, Armonk, New York, USA). Results of quantitative variables analyses are expressed as means \pm standard deviations, whereas results of categorical variables are expressed as counts and percentages. Association between categorical variables was assessed using Fisher's exact test. The Gaussian distribution of continuous variables was verified with the Kolmogorov-Smirnov test. Comparisons of mean age, BCVA, and axial length between patients with and without m-MNV were performed using Student's independent samples $t$ test. Comparisons of mean BCVA between baseline and the end of follow-up were performed using the Student paired $t$ test. In all analyses, $p$ values less than 0.05 were considered statistically significant.

\section{RESULTS}

Among 33 eyes of 33 consecutive patients affected by macular hemorrhage secondary to $\mathrm{MM}, 14$ eyes of 14 patients fulfilled the inclusion and exclusion criteria and were included in the study. Fourteen cases were excluded because of the lack of one of the two required angiographic examinations (i.e., FA or OCTA). On the other hand, five patients were excluded because of the poor quality of the OCT or OCTA images.

The mean age was $60 \pm 16$ years (median 59; range 33-85 years) and all patients were Caucasian. Thirteen patients were female, and one patient was male. Mean axial length was $27.4 \pm 0.8 \mathrm{~mm}$ (range $26.4-28.5 \mathrm{~mm}$ ). Nine out of 14 eyes (64\%) were phakic and $5(36 \%)$ were pseudophakic. Myopic posterior staphylomas were noted in six eyes and were categorized on the basis of Ohno-Matsui's classification [20], as follows: one eye presented a type I staphyloma, three eyes a type II staphyloma, and two eyes a type III staphyloma. In accordance with the exclusion criteria, all eyes displayed no other retinal diseases besides MM. No dome-shaped macula aspect was identified in the eyes considered. 

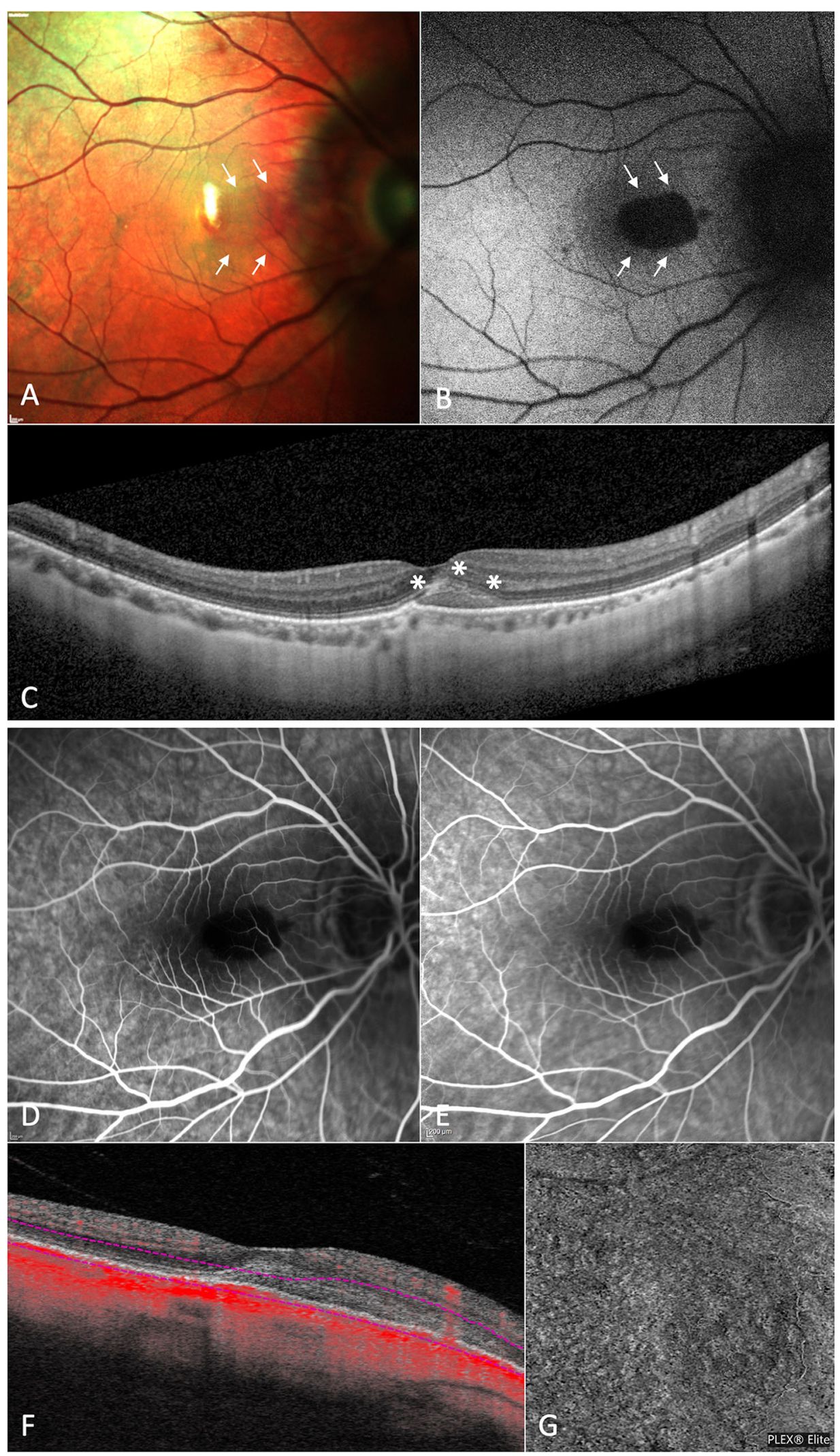
4Fig. 2 Multimodal retinal imaging of a patient with high myopia affected by macular hemorrhage due to spontaneous Bruch's membrane rupture in the right eye (i.e., idiopathic macular hemorrhage). Multicolor imaging (a) and fundus autofluorescence (b) showing the presence of a macular hemorrhage involving the subfoveal area (white arrows). Horizontal structural optical coherence tomography (OCT) passing through the fovea (c) illustrating the presence of subretinal hyperreflective material (SHRM) above the retinal pigment epithelium layer without subretinal and/or intraretinal hyporeflective exudation (asterisks). Fluorescein angiography displaying a hypofluorescent area due to a masking effect in the site of the macular hemorrhage in both early and late phases of examination (d and e, respectively). $6 \times 6 \mathrm{~mm}$ en-face OCT-angiography image (g) and corresponding B-scans with flow (f) excluding the presence of anomalous flow in the SHRM location

At baseline (i.e., time of macular hemorrhage diagnosis) mean BCVA was between 20/50 and 20/63 Snellen equivalent $(0.46 \pm 0.33 \log \mathrm{MAR}$; median, 0.35; range 0.20-1.30 logMAR). On the basis of fundus examination recordings, fundus color pictures, and/or multicolor imaging, macular hemorrhage was located in the subfoveal area in 7 out of 14 cases (50\%), in the parafoveal area in 4 out of 14 cases (29\%), and in the extrafoveal area in 3 out of 14 cases (21\%).

By means of structural OCT, all hemorrhages appeared as subretinal hyperreflective material (SHRM) above the RPE layer (Figs. 1 and 2). In 8 out of 14 cases $(57 \%)$, FA displayed well-defined hyperfluorescence in the early angiographic phases with moderate leakage in the late phases, matching with a diagnosis of type $2 \mathrm{MNV}$ (Fig. 1). On the other hand, the remaining 6 cases (43\%) showed no hyperfluorescence by means of FA, matching with a diagnosis of IMH (Fig. 2). Of note, in all cases, FA displayed a hypofluorescent area due to a masking effect in the site of the macular hemorrhage.

OCTA was performed on the same day as FA examination. Interestingly, OCTA displayed the presence of a neovascular network in all cases previously diagnosed as type $2 \mathrm{MNV}$ using FA. Furthermore, OCTA also excluded the presence of anomalous flow in all eyes without signs of $\mathrm{m}-\mathrm{MNV}$ by means of FA. This accounted for the high sensitivity and specificity of OCTA for m-MNV detection in HM cases with macular hemorrhage. Demographics and main clinical features of patients affected by $\mathrm{m}-\mathrm{MNV}$ and IMH are reported in Table 1.

According to inclusion and exclusion criteria, all cases were revaluated after 3 months. All eight cases affected by $\mathrm{m}-\mathrm{MNV}$ were treated by a mean of $2.3 \pm 0.9$ (range 1-3) intravitreal injections of anti-VEGF using a pro re nata (PRN) regimen. After the treatment, the macular hemorrhage was reabsorbed in all cases, and BCVA significantly improved from $0.39 \pm 0.15 \log \mathrm{MAR}$ to $0.21 \pm 0.14 \log \mathrm{MAR}$ $(p=0.006)$. On the other hand, patients affected by IMH were followed without any treatment. At the end of the follow-up, all six patients affected by IMH displayed reabsorption of the macular hemorrhage and an improvement of BCVA from $0.55 \pm 0.48 \log$ MAR to $0.17 \pm 0.08 \log \mathrm{MAR}(p=0.112)$.

\section{DISCUSSION}

In the present study, we reported that OCTA was able to discern between macular hemorrhage due to $\mathrm{m}-\mathrm{MNV}$ and due to spontaneous Bruch's membrane rupture in all the $14 \mathrm{HM}$ eyes presenting with fundoscopic evidence of focal macular hemorrhage.

Subretinal bleeding is a common finding in $\mathrm{HM}$ eyes. Besides the rare cases of eye trauma, the most common sources are m-MNV and IMH. Population studies reported a prevalence of m-MNV complicating HM of between 5.2\% and $11.3 \%$ [10]. Bruch's membrane rupture, commonly seen in deep myopic staphyloma and lacquer crack, or large patches of retinal atrophy could lead to an impaired balance between VEGF and anti-angiogenic factors, ultimately leading to m-MNV formation [21]. Conversely, IMH results from focal spontaneous Bruch's membrane rupture with the prompt passage of a small amount of blood into the subretinal space. In this sense, it is speculated that IMH could be an early sign of new lacquer crack formation [22]. However, the pathophysiological processes are not always independent and overlapping could occur. For example, the 
role of perforating vessels is emblematic: their expansion in the scleral tissue seems to be the primary cause of lacquer cracks, but also a recognized risk factor for MNV [6]. Indeed, Giuffrè et al. [23] found a high prevalence $(70 \%)$ of perforating vessels beneath $\mathrm{m}-\mathrm{MNV}$ by means of structural OCT, confirming the previous assertion. At the same time, the mechanical stretch determined by perforating vessels is the major cause of IMH [24].

In the case of macular bleeding, the clinical distinction between IMH and m-MNV is of paramount importance for therapy and prognosis. The former has a scarce impact on the visual outcome and enables a wait-and-see approach until complete blood reabsorption. On the other hand, the latter needs prompt treatment, based on intravitreal anti-VEGF therapy, and a regular follow-up for an early diagnosis of relapses [25]. However, discerning the origin of the subretinal bleeding is not always predictable, even with the most sophisticated imaging technique.

FA is still considered the gold standard in confirming neovascularization in MM [26]. Characteristic features on FA are those of a type $2 \mathrm{MNV}$ : well-defined hyperfluorescence in the early angiographic phase with moderate leakage in the late phases. Nonetheless, FA has several important shortcomings. FA is an invasive procedure and adverse events, even serious ones such as anaphylactic shock, must be taken into account. From an interpretative point of view, an extensive retinal hemorrhage could block the underneath fluorescence, partially or totally masking the neovascularization and causing failure of the diagnostic process [27].

In this study, we observed that OCTA could be considered as a reliable choice in identifying a neovascular pattern in cases of myopic hemorrhages, thus differentiating m-MNV from an IMH with high sensitivity and specificity.

The clinical importance is indisputable: a repeatable and fast-acquiring imaging tool could provide essential data to the clinician, avoiding an invasive and potentially harmful examination of the patient. In this sense, our data are in agreement with previous reports from the literature. OCTA, when available, is a feasible alternative for the detection of $\mathrm{m}-\mathrm{MNV}$.
Table 1 Main clinical features at baseline of patients with high myopia affected by macular hemorrhages $(\mathrm{MH})$ due to myopic macular neovascularization (m-MNV) or by idiopathic $\mathrm{MH}$ by means of fluorescein angiography

\begin{tabular}{|c|c|c|c|}
\hline & $\begin{array}{l}\text { m-MNV } \\
(n=8)\end{array}$ & $\begin{array}{l}\text { Idiopathic } \\
\text { MH }(n=6)\end{array}$ & $p$ value \\
\hline $\begin{array}{l}\text { Age, years } \\
\qquad(\text { mean } \pm S D)\end{array}$ & $64 \pm 17$ & $53 \pm 14$ & $0.218^{\mathrm{a}}$ \\
\hline $\begin{array}{c}\text { BCVA, logMAR } \\
(\text { mean } \pm \text { SD })\end{array}$ & $0.39 \pm 0.15$ & $0.55 \pm 0.48$ & $0.376^{\mathrm{a}}$ \\
\hline $\begin{array}{l}\text { Axial length, } \\
\text { mm } \\
(\text { mean } \pm S D)\end{array}$ & $27.17 \pm 0.82$ & $27.62 \pm 0.66$ & $0.292^{\mathrm{a}}$ \\
\hline $\begin{array}{l}\text { Posterior } \\
\text { staphyloma }\end{array}$ & $2(1 \mathrm{I} ; 1 \mathrm{II})^{\mathrm{c}}$ & $\begin{array}{c}4(2 \mathrm{II} ; 2 \\
\text { III) })^{\mathrm{c}}\end{array}$ & \\
\hline $\begin{array}{l}\text { Localization of } \\
\mathrm{MH}, n(\%)\end{array}$ & & & $0.500^{\mathrm{b}}$ \\
\hline Subfoveal & $4(50 \%)$ & $3(50 \%)$ & \\
\hline Parafoveal & $3(37 \%)$ & $1(17 \%)$ & \\
\hline Extrafoveal & $1(13 \%)$ & $2(33 \%)$ & \\
\hline $\begin{array}{l}\text { Neovascular } \\
\text { network using } \\
\text { OCTA, } n(\%)\end{array}$ & $8(100 \%)$ & $6(100 \%)$ & \\
\hline
\end{tabular}

$n$ number, $S D$ standard deviation, $B C V A$ best-corrected visual acuity, OCTA optical coherence tomography angiography

${ }^{a}$ Student's independent samples $t$ test

${ }^{\mathrm{b}}$ Fisher's exact test

'According to Ohno-Matsui's classification for myopic staphyloma

Our study results are consistent with the report from Miyata et al. in which a detection rate of 94.1\% was assessed using OCTA alone in type $2 \mathrm{~m}-\mathrm{MNV}$ [28]. Interestingly, even smallsized $\mathrm{m}-\mathrm{MNV}$ (range $0.22 \pm 0.27 \mathrm{~mm}^{2}$ ) can be successfully revealed [29]. In a cohort of 36 eyes, Querques et al. confirmed that OCTA was able to identify myopic MNVs in 19 out of 21 eyes, and correctly excluded 30 out of 32 eyes with pathological myopia without MNV (sensitivity was $90.48 \%$ and specificity $93.75 \%$ ) [30]. However, previous studies did not evaluate the 
diagnostic capacity of OCTA in the differential diagnosis of macular myopic hemorrhages. Our data suggest that not only can OCTA clearly identify m-MNVs clinically presenting with a hemorrhage but it can also exclude neovascularization in the case of IMH, adding an additional clinical role to this imaging technique.

As stated by previous articles, OCTA technology has several limitations in its application to myopic eyes: the high axial length, the frequent fixation instability, and the morphologic atypical structure (i.e., staphyloma, scleral thinning) that could lead to poor quality or a non-perfectly segmented image $[31,32]$. This is a limitation in the application in the clinical setting of OCTA. However, we feel that a good, focused, and patiently repeated OCTA examination, even with adjusted post-acquisition manual segmentation, could avoid invasive imaging of the patient, something which is not free from complications. In the future, higher speed instruments and ameliorated focus might be able to overcome the actual technical limits of the instruments.

The major limitation of this study is the relatively low sample size. However, we included only patients with HM affected by macular hemorrhage that underwent both imaging analyses (i.e., FA and OCTA) on the same day at the time of the diagnosis. Furthermore, the extremely good accordance between OCTA and fluorescein angiography results is a considerable point. In addition, a female predominance in our sample could have determined a bias leading to our results not being entirely generalizable. Finally, we recognize that indocyanine green angiography (ICGA) is a useful complementary exam in discerning between $\mathrm{m}-\mathrm{MNV}$ and IHM; however, ICGA data in our cohort were very scarce and therefore were excluded.

\section{CONCLUSION}

This study confirms that OCTA is able to discern with excellent reliability between the presence of a neovascular network in patients with $\mathrm{HM}$ presenting with a new macular hemorrhage and simple bleeding due to spontaneous Bruch's membrane rupture.

\section{ACKNOWLEDGEMENTS}

We thank the participants of the study.

Funding. No funding or sponsorship was received for this study or publication of this article.

Authorship. All the named authors meet the International Committee of Medical Journal Editors (ICMJE) criteria for authorship for this article and take responsibility for the integrity of the work as a whole. All the authors have given their approval for this version to be published.

Author Contributions. Giuseppe Querques, Marco Battista and Riccardo Sacconi conceived the concept and design of the paper. Riccardo Sacconi contributed to the statistical analysis and Marco Battista, Riccardo Sacconi, Enrico Borrelli, Anna Crepaldi and Federico Fantaguzzi to drafting the manuscript. Giuseppe Querques, Francesco Bandello, Riccardo Sacconi, Mariacristina Parravano, Daniele de Geronimo, Eliana Costanzo and Enrico Borrelli supervised and made the final revision.

Disclosures. Marco Battista, Anna Crepaldi, Federico Fantaguzzi, Eliana Costanzo, Daniele de Geronimo: none.Riccardo Sacconi and Enrico Borrelli are consultants for Novartis (Basel, Switzerland), and Zeiss (Dublin, USA).Mariacristina Parravano has the following disclosures: Allergan Inc (Irvine, California,USA) Bayer Shering-Pharma (Berlin, Germany), Novartis (Basel, Switzerland).Francesco Bandello is a consultant for Alcon (Fort Worth, Texas, USA), Alimera Sciences (Alpharetta, Georgia, USA), Allergan Inc (Irvine, California,USA), Farmila-Thea (Clermont-Ferrand, France), Bayer Shering-Pharma (Berlin, Germany), Bausch and Lomb (Rochester, New York, USA), Genentech (San Francisco, California, USA), Hoffmann-La-Roche (Basel, Switzerland), NovagaliPharma (Évry, France), Novartis (Basel, Switzerland), Sanofi-Aventis (Paris, France), Thrombogenics (Heverlee,Belgium), Zeiss (Dublin, USA).Giuseppe Querques is a consultant for Alimera Sciences (Alpharetta, Georgia, 
USA), Allergan Inc (Irvine, California,USA), Amgen (Thousand Oaks, USA), Heidelberg (Germany), KBH (Chengdu, China), LEH Pharma (London, UK), Lumithera (Poulsbo, USA), Novartis (Basel, Switzerland), Bayer Shering-Pharma (Berlin, Germany), Sandoz (Berlin, Germany), Sifi (Catania, Italy), Soof-Fidia (Albano, Italy), Zeiss (Dublin, USA).Other Acknowledgments: The PLEX ® Elite 9000 has been made available through the Advanced Retina Imaging Network for which Giuseppe Querques is a steering committee member.

Compliance with Ethics Guidelines. This double-center observational study of patients affected by macular hemorrhages in high myopia, conducted in the Ophthalmology Department of San Raffaele Hospital, Milan, Italy and G. B. Bietti Foundation, Rome, Italy, adhered to the Helsinki declaration and its later amendments. The local institutional review board was informed and approved the retrospective review of data.

Data Availability. All data generated or analyzed during this study are included in this published article.

Open Access. This article is licensed under a Creative Commons Attribution-NonCommercial 4.0 International License, which permits any non-commercial use, sharing, adaptation, distribution and reproduction in any medium or format, as long as you give appropriate credit to the original author(s) and the source, provide a link to the Creative Commons licence, and indicate if changes were made. The images or other third party material in this article are included in the article's Creative Commons licence, unless indicated otherwise in a credit line to the material. If material is not included in the article's Creative Commons licence and your intended use is not permitted by statutory regulation or exceeds the permitted use, you will need to obtain permission directly from the copyright holder. To view a copy of this licence, visit http://creativecommons.org/licenses/by$\mathrm{nc} / 4.0 /$.

\section{REFERENCES}

1. Ruiz-Medrano J, Montero JA, Flores-Moreno I, Arias L, García-Layana A, Ruiz-Moreno JM. Myopic maculopathy: current status and proposal for a new classification and grading system (ATN). Prog Retin Eye Res. 2019. https://doi.org/10.1016/j.preteyeres. 2018.10.005.

2. Curtin BJ, Karlin DB. Axial length measurements and fundus changes of the myopic eye. Am J Ophthalmol. 1971. https://doi.org/10.1016/00029394(71)91092-0.

3. Ohno-Matsui K, Kawasaki R, Jonas JB, et al. International photographic classification and grading system for myopic maculopathy. Am J Ophthalmol. 2015. https://doi.org/10.1016/j.ajo.2015.01.022.

4. Wong TY, Ohno-Matsui K, Leveziel N, et al. Myopic choroidal neovascularisation: current concepts and update on clinical management. Br J Ophthalmol. 2015. https://doi.org/10.1136/bjophthalmol-2014305131.

5. Ikuno $\mathrm{Y}$, Jo Y, Hamasaki T, Tano Y. Ocular risk factors for choroidal neovascularization in pathologic myopia. Investig Ophthalmol Vis Sci. 2010. https://doi.org/10.1167/iovs.09-3493.

6. Querques G, Corvi F, Balaratnasingam C, et al. Lacquer cracks and perforating scleral vessels in pathologic myopia: a possible causal relationship. Am J Ophthalmol. 2015. https://doi.org/10.1016/j. ajo.2015.07.017.

7. Ohno-Matsui K, Lai TYY, Lai CC, Cheung CMG. Updates of pathologic myopia. Prog Retin Eye Res. 2016. https://doi.org/10.1016/j.preteyeres.2015.12. 001.

8. Ren P, Lu L, Tang X, et al. Clinical features of simple hemorrhage and myopic choroidal neovascularization associated with lacquer cracks in pathologic myopia. Graefes Arch Clin Exp Ophthalmol. 2020. https://doi.org/10.1007/s00417-020-04778-6.

9. Franqueira N, Cachulo ML, Pires I, et al. Long-term follow-up of myopic choroidal neovascularization treated with ranibizumab. Ophthalmologica. 2011. https://doi.org/10.1159/000333213.

10. Cheung CMG, Arnold JJ, Holz FG, et al. Myopic choroidal neovascularization: review, guidance, and consensus statement on management. Ophthalmology. 2017. https://doi.org/10.1016/j. ophtha.2017.04.028.

11. Battista M, Borrelli E, Parravano $\mathrm{M}$, et al. OCTA characterisation of microvascular retinal alterations in patients with central serous chorioretinopathy. 
Br J Ophthalmol. 2019. https://doi.org/10.1136/ bjophthalmol-2019-315482.

12. Carnevali A, Sacconi R, Querques L, et al. Natural history of treatment-naïve quiescent choroidal neovascularization in age-related macular degeneration using OCT angiography. Ophthalmol Retin. 2018. https://doi.org/10.1016/j.oret.2018.02.002.

13. Carnevali A, Capuano V, Sacconi R, et al. OCT angiography of treatment-naïve quiescent choroidal neovascularization in pachychoroid neovasculopathy. Ophthalmol Retin. 2017. https://doi. org/10.1016/j.oret.2017.01.003.

14. Sacconi R, Battista M, Borrelli E, et al. OCT-A characterisation of recurrent type 3 macular neovascularisation. Br J Ophthalmol. 2020. https://doi. org/10.1136/bjophthalmol-2020-316054.

15. Sacconi R, Sarraf D, Garrity S, et al. Nascent type 3 neovascularization in age-related macular degeneration. Ophthalmol Retin. 2018. https://doi.org/10. 1016/j.oret.2018.04.016.

16. Sacconi R, Tomasso L, Corbelli E, et al. Early response to the treatment of choroidal neovascularization complicating central serous chorioretinopathy: a OCT-angiography study. Eye. 2019. https://doi.org/10.1038/s41433-019-0511-2.

17. Gong J, Yu S, Gong Y, Wang F, Sun X. The diagnostic accuracy of optical coherence tomography angiography for neovascular age-related macular degeneration: a comparison with fundus fluorescein angiography. J Ophthalmol. 2016. https://doi. org/10.1155/2016/7521478.

18. Kedhar SR, Thorne JE, Wittenberg S, Dunn JP, Jabs DA. Multifocal choroiditis with panuveitis and punctate inner choroidopathy: comparison of clinical characteristics at presentation. Retina. $2007 . \quad$ https://doi.org/10.1097/IAE. Ob013e318068de72.

19. Huang Y, Gangaputra S, Lee KE, et al. Signal quality assessment of retinal optical coherence tomography images. Invest Ophthalmol Vis Sci. 2012. https:// doi.org/10.1167/iovs.11-8755.

20. Ohno-Matsui K, Jonas JB. Posterior staphyloma in pathologic myopia. Prog Retin Eye Res. 2019. https://doi.org/10.1016/j.preteyeres.2018.12.001.

21. Ohno-Matsui K, Jonas JB, Spaide RF. Macular bruch membrane holes in highly myopic patchy chorioretinal atrophy. Am J Ophthalmol. 2016. https:// doi.org/10.1016/j.ajo.2016.03.019.

22. Ohno-Matsui K, Ito M, Tokoro T. Subretinal bleeding without choroidal neovascularization in pathologic myopia: a sign of new lacquer crack formation. Retina. 1996. https://doi.org/10.1097/ 00006982-199616030-00003.

23. Giuffrè C, Querques L, Carnevali A, De Vitis LA, Bandello F, Querques G, et al. Choroidal neovascularization and coincident perforating scleral vessels in pathologic myopia. Eur J Ophthalmol. 2017. https://doi.org/10.5301/ejo.5000875.

24. Liu B, Zhang X, Mi L, Chen L, Wen F. Long-term natural outcomes of simple hemorrhage associated with lacquer crack in high myopia: a risk factor for myopic CNV? J Ophthalmol. 2018. https://doi.org/ $10.1155 / 2018 / 3150923$.

25. Parodi MB, Iacono P, Sacconi R, Iuliano L, Bandello F. Fundus autofluorescence changes after ranibizumab treatment for subfoveal choroidal neovascularization secondary to pathologic myopia. Am J Ophthalmol. 2015. https://doi.org/10.1016/j.ajo. 2015.04.030.

26. Leveziel N, Caillaux V, Bastuji-Garin S, Zmuda M, Souied EH. Angiographic and optical coherence tomography characteristics of recent myopic choroidal neovascularization. Am J Ophthalmol. 2013. https://doi.org/10.1016/j.ajo.2012.11.021.

27. Reichel E, Duker JS, Puliafito CA. Indocyanine green anglography and choroidal neovascularization obscured by hemorrhage. Ophthalmology. 1995. https://doi.org/10.1016/S0161-6420(95)30781-6.

28. Miyata M, Ooto S, Hata $M$, et al. Detection of myopic choroidal neovascularization using optical coherence tomography angiography. Am J Ophthalmol. 2016. https://doi.org/10.1016/j.ajo.2016. 03.009 .

29. Bruyère E, Miere A, Cohen SY, et al. Neovascularization secondary to high myopia imaged by optical coherence tomography angiography. Retina. 2017. https://doi.org/10.1097/IAE.0000000000001456.

30. Querques L, Giuffrè C, Corvi F, et al. Optical coherence tomography angiography of myopic choroidal neovascularisation. $\mathrm{Br} \mathrm{J}$ Ophthalmol. 2017. https://doi.org/10.1136/bjophthalmol-2016309162.

31. Spaide RF, Fujimoto JG, Waheed NK, Sadda SR, Staurenghi G. Optical coherence tomography angiography. Prog Retin Eye Res. 2018. https://doi. org/10.1016/j.preteyeres.2017.11.003.

32. Iacono $\mathrm{P}$, Giorno $\mathrm{P}$, Varano $\mathrm{M}$, Parravano $\mathrm{M}$. Structural and optical coherence tomography angiography in myopic choroidal neovascularization: agreement with conventional fluorescein angiography. Eur J Ophthalmol. 2021. https://doi. org/10.1177/1120672119882333. 